Ferdinand Z. Guintu, MD Alexander T. Laoag, MD Joselito F. David, MD

Department of Otorhinolaryngology Head and Neck Surgery

Jose R. Reyes Memorial Medical Center
Correspondence: Dr. Joselito F. David Department of Otorhinolaryngology - Head and Neck Surgery Jose R. Reyes Memorial Medical Center San Lazaro Compound, Rizal Avenue

Sta. Cruz, Manila 1003

Philippines

Phone: (632) 743 6921; (632) 7119491 local 320

Email:entjrrmmc@yahoo.com

Reprints will not be available from the authors.

The authors declared that this represents original material that is not being considered for publication or has not been published or accepted for publication elsewhere, in full or in part, in print or electronic media; that the manuscript has been read and approved by all the authors, that the requirements for authorship have been met by each author, and that each author believes that the manuscript represents honest work.

Disclosures: The authors signed disclosures that there are no financial or other (including personal) relationships, intellectual passion, political or religious beliefs, and institutional affiliations that might lead to a conflict of interest.

Presented at: Interesting Case Contest, Philippine Society of Otolaryngology Head and Neck Surgery, lloilo Grand Hotel, Iloilo City, Philippines, April 28, 2012.

\title{
Gap Arthroplasty of Bilateral Temporomandibular Joint Ankylosis
}

\begin{abstract}
Objectives: To present a case of bilateral temporomandibular joint ankylosis that was managed successfully through gap arthroplasty.
\end{abstract}

\section{Methods:}

Design: Case Report

Setting: Tertiary Government Hospital

Patient: One

Results: A 25-year-old man presented with inability to open his mouth for 18 years after direct trauma to his chin. CT scan showed bilateral bony fusion of condyles to glenoid fossae, hypertrophic sclerosis and fusion of the condylar heads to the temporal bones. He underwent bilateral gap arthroplasty via preauricular approach with creation of a $15 \mathrm{~mm}$ space on the mandibular fossa. As of latest follow up, the patient maintained an inter-alveolar distance of $30 \mathrm{~mm}$ for five months postoperatively through continuous aggressive mouth opening exercises.

Conclusion: Gap arthroplasty may be an efficient procedure for temporomandibular joint ankylosis in achieving satisfactory post-operative inter-alveolar opening and articular function. Early and meticulous rehabilitation is required to prevent relapse. Long-term follow up is recommended to document possible recurrence.

Keywords: temporomandibular joint ankylosis, gap arthroplasty, TMJ ankylosis, ankylosis

Temporomandibular joint (TMJ) ankylosis is the union of articular surfaces (mandibular condyle to the cranial base) by means of osseous and/or fibrous tissue with partial or complete mandibular impediment. ${ }^{1}$ The most common etiologic factors include trauma (13-100\%), infections (10-49\%), rheumathoid arthritis (10\%), congenital anomalies and neoplastic processes. ${ }^{2}$

It is a condition leading to problems in mastication, digestion, speech, facial and oral hygiene. When acquired at childhood, devastating effects are observed during growth and development of teeth and jaws. It can negatively influence the psychosocial behavior of the patient due to the consequent facial deformity magnified as the child grows. ${ }^{2}$ Various methods have been used to manage TMJ ankylosis including gap arthroplasty, interpositional arthroplasty and joint reconstruction by bone grafts or joint prosthesis. ${ }^{1,2,3}$ Recent studies advocate distraction osteogenesis in management of TMJ ankylosis as it provides excellent cosmetic results. ${ }^{4}$

A local report in 1984 by Nolasco et al. involved a case of bilateral TMJ ankylosis treated with interpositional arthroplasty wherein $5 \mathrm{~mm}$ length of the bone was removed from edge to edge then interposed with temporalis fascia and muscle. ${ }^{4}$ The patient 
failed to follow up two weeks postoperatively and no further documentation of success or recurrence could be made. ${ }^{4}$

Gap arthroplasty can be an additional armamentarium for practicing otolaryngologists in developing countries where a major reason for delayed management is the lack of adequate resources. Our goal was to perform a simple yet effective procedure amenable to our Filipino patients. We present a case of bilateral temporomandibular joint ankylosis that was managed successfully through gap arthroplasty. To the best of our knowledge, this is the first such case documented in the Philippines.

\section{CASE REPORT}

A 25-year-old man consulted due to inability to open his mouth. At the age of 7 years, he hit his chin on the handlebar of a jeepney during a vehicular accident. He was admitted with multiple contusions and discharged on the fifth hospital day. A week after discharge, he experienced gradual and progressive limitation of jaw movement. He consulted his attending physician and was advised that the limitation of jaw movement was due to infection. Antibiotics did not improve his symptoms and he ceased to follow-up. He had progressive loss of jaw movement but was able to feed himself by inserting shredded pieces of noodles and meat through the small space between his teeth.

Physical examination revealed a classical 'bird face appearance." (Figure 1A) There was excessive vertical overlap of the maxillary incisors (overbite), horizontal extension anteriorly of the maxillary incisors (overjet), dental caries and periodontal disease. Mouth opening is measured via inter-incisal distance but for edentulous cases, inter-alveolar distance is used. Our patient had zero inter-alveolar distance. (Figure 1B) There were no gliding, protrusion and lateral movements of the mandible.

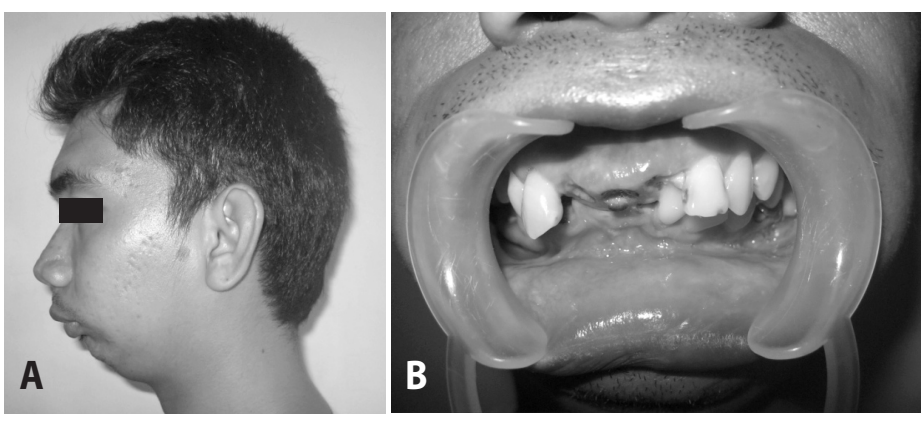

Figures 1A. Retrognathia "bird face" appearance; B. Inter-alveolar distance of zero

Facial CT scans revealed bilateral bony fusion of the condyles to the glenoid fossae, hypertrophic sclerosis with fusion of the condylar heads to the temporal bones and shortening of the mandibular bodies with inward displacement of the symphysis mentum. (Figure 2)

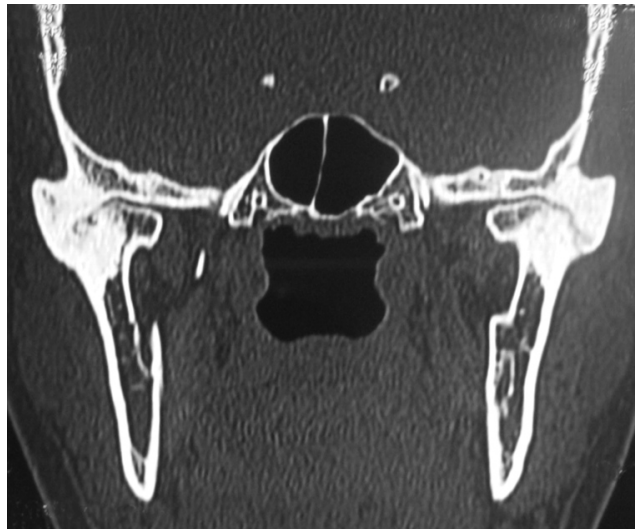

Figure 2. Hypertrophic sclerosis and fusion of the lateral aspect of condylar heads to the temporal bone.
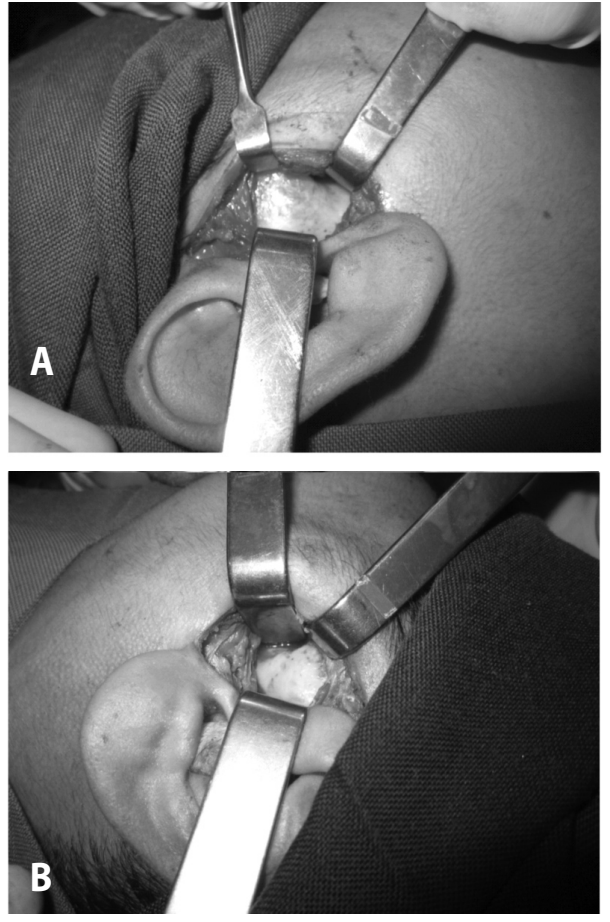

Figures 3 A \& B. Intraoperative finding of bilateral bony fusion of condyle and coronoid on the temporal bone

After extensive literature review of the pros and con of the three commonly used treatment modalities for TMJ ankylosis, we decided on gap arthroplasty. It is cheap, yet effective in achieving desired mouth opening, less invasive with no donor site morbidity. The operation was explained to the patient and he consented to the surgery. Under general anesthesia via tracheostomy, both TMJ were exposed through a preauricular approach taking care to preserve the branches of the facial 
nerve. After exposure and identification of the sites of ankylosis (Figure 3), the fibrous and bony masses were aggressively excised with round burs and chisels until adequate mandibular movements were achieved. Bilateral coronoidectomies were also performed. A gap of $15 \mathrm{~mm}$ was created between the recontoured glenoid fossa and mandible. (Figure 4) The cut sharp edges were smoothened with diamond burs.
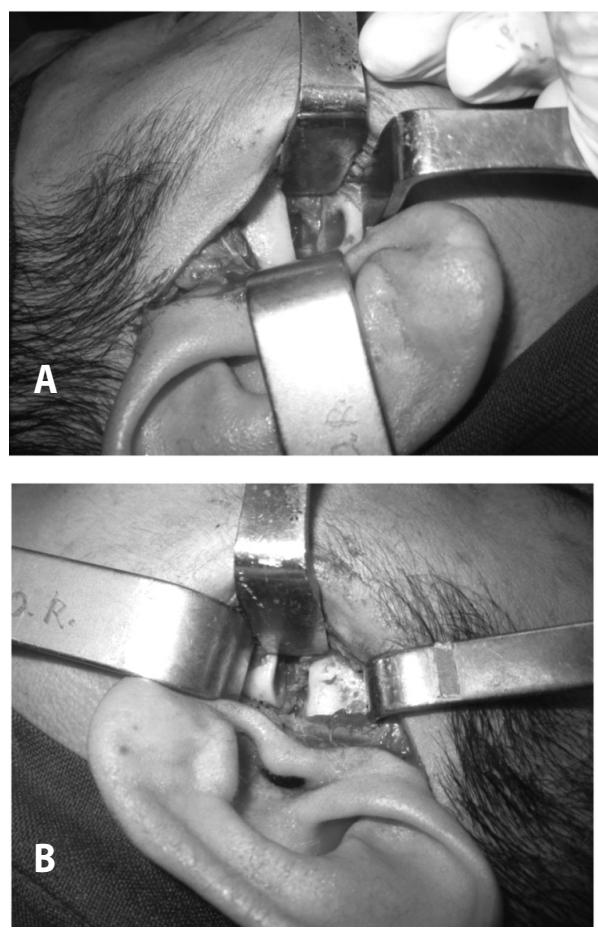

Figures 4A \& B. A $15 \mathrm{~mm}$ space created on bilateral mandibular fossa

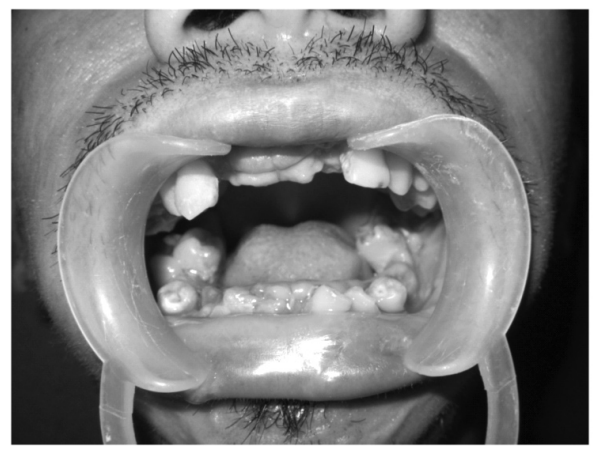

Figure 5. One week postoperative inter-alveolar distance of $30 \mathrm{~mm}$

One week postoperatively, the patient had an inter-alveolar distance of $30 \mathrm{~mm}$. (Figure 5) Physiotherapy included stretching and electrical stimulation of muscles of mastication and the contract-hold-relax technique for masticatory muscles. At five months post-physiotherapy, he had gained weight and was well satisfied with the operation. Inter-alveolar distance then remained at $30 \mathrm{~mm}$. (Figure 6)

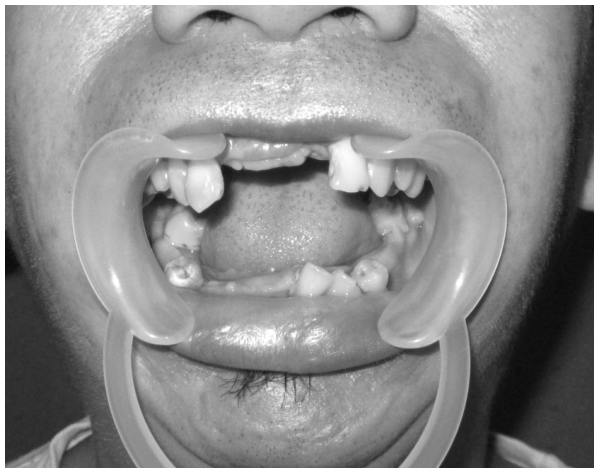

Figure 6. A 30mm interalveolar distance 5 months post operatively

\section{DISCUSSION}

Ankylosis of the TMJ is a challenging problem for both the patient and surgeon. Over the years, fundamental principles of TMJ surgery and "trial and error" have shaped the evolution of different techniques to correct the problem. ${ }^{6}$

In this case, trauma directed to the patient's chin led to bilateral condylar fractures. Neglected condylar fractures resulted in TMJ ankylosis.

TMJ ankylosis is classified according to location (intra or extraarticular), type of tissue (osseous, fibrous, or fibro-osseous) and the degree of union (partial or complete) involved. ${ }^{1}$ It is further classified into true and false ankylosis. True ankylosis results in osseous or fibrous adhesion between the surfaces of the TMJ, while false ankylosis results from diseases not directly related to the joint. Sawhney in 1986 classified TMJ ankylosis into four different types: Type I- presence of fibroadhesions at the condyle; Type II - bone fusion with condyle remodeling and an intact medial pole; Type III - anquilotic mass, mandibular ramus union with the zygomatic arch and medial pole intact; and Type IV - complete anquilotic mass, total union of the mandibular ramus with the zygomatic arch.' Our patient's TMJ ankylosis was classified as intra-articular, fibroosseus, partial union and type II (only the lateral aspect has bony fusion).

The diagnostic process consists of physical and radiographic examinations (CT with three-dimensional (3D) reconstruction). Radiographic findings include condylar deformation, narrowing or irregularities at the inter-articular space. Early diagnosis and treatment are pivotal to avoid sequelae. ${ }^{3}$ In our case, financial constraints wasted 18 years without treatment. The best results can be achieved after complete evaluation and establishment of long-term treatment planning.

A variety of treatment techniques have been described in the literature. The three most commonly used are gap arthroplasty, interpositional arthroplasty and excision of ankylosed bone with articular reconstruction. ${ }^{1,2,3}$ Vasconcelos et al. reported 


\section{CASE REPORTS}

eight cases of TMJ ankylosis managed by gap arthroplasty with no recurrence within a 24-month period. ${ }^{2}$

There is still no agreed standard treatment for TMJ ankylosis. Results of frequently reported operations like gap arthroplasty, interpositional arthroplasty and joint reconstruction with autogenous or alloplastic materials have been variable and often less than satisfactory because of documented recurrences. The interposition of autogenous or alloplastic materials at the osteotomy site may lead to morbidity at the donor site, unpredictable resorption and risk of a foreign body reaction. ${ }^{6}$ Gap arthroplasty is a frequently used surgical option that is less invasive and requires less surgical time. The postoperative condition is more comfortable because no donor site is required, reducing the risk of lesions to other structures. The recurrence rate for gap arthroplasty is $13 \%$. Relapse usually occurred in Sawhney type IV ankylosis. Aggressive physiotherapy after surgery will reduce recurrence. ${ }^{2,6}$

Meticulous preoperative planning, perioperative management and diligent postoperative care remain the keys to successful surgery. Gap arthroplasty may be an efficient procedure for temporomandibular joint ankylosis in achieving satisfactory post-operative inter-alveolar opening and articular function. Early and meticulous rehabilitation is required to prevent relapse. Long-term follow up is recommended to document possible recurrence.

\section{REFERENCES}

1. de Andrade LH, Cavalcante MA, Raymundo R Jr, de Souza IP. Temporomandibular joint ankylosis in children. J Dent. Child. 2009 Jan-Apr; 76(1): 41-5.

2. Vasconcelos BC, Porto GG, Bessa-Noguiera RV, Nascimento MM. Surgical treatment of temporomandibular joint ankylosis: follow-up of 15 cases and literature review. Med Oral Patol Oral Cir Bucal. 2009 Jan 1; 14(1): 34-8.

3. Balaji SM. Modified temporalis anchorage in craniomandibular reankylosis. Int J Oral Maxillofac Surg. 2003 Oct; 32(5): 480-5.

4. Mehrotra D, Dhasmanaa S, Kumar S. Management of temporomandibular ankylosis with temporal fascia inter-positional arthroplasty and distraction osteogenesis: report of 30 cases. J Long Term Eff Med Implants. 2009;19 (2):139-48.

5. Nolasco F, Cosalan E, dela Cruz R. Bilateral ankylosis of the temporomandibular joint. Philipp J Otolaryngol Head Neck Surg. 1984: 309-11.

6. Felstead AM, Revington PJ. Surgical management of temporomandibular joint ankylosis in ankylosing spondylitis. Int J Rheumatol. 2011 Jan; 2011: 854167.

7. Sawhney CP. Bony ankylosis of the temporomandibular joint: follow-up of 70 patients treated with arthroplasty and acrylic spacer interposition. Plast. Reconstr. Surg. 1986 Jan; 77(10): 29-40. 